\title{
The Incidence of Multiplicity Among Bright Stellar Systems
}

\author{
Peter P. Eggleton ${ }^{1}$, Ludmila Kisseleva-Eggleton ${ }^{2}$ \\ and Xander Dearborn ${ }^{3}$ \\ ${ }^{1}$ Institute of Geophysics and Planetary Physics, \\ Lawrence Livermore National Laboratory, 7000 East Ave, Livermore, CA94551, USA \\ email: ppe@igpp.ucllnl.org \\ ${ }^{2}$ U. C. Berkeley Extension, 1995 University Ave, Berkeley, CA 94720-7002 \\ email: ludmilake@yahoo.com \\ ${ }^{3} 1770$ Walnut Ave, Livermore, CA 94551, USA \\ email: powerhawk56@hotmail.com
}

\begin{abstract}
We consider the multiplicity of stellar systems with (combined) magnitude brighter than 6.00 in Hipparcos magnitudes. We identify 4555 such bright systems, and the frequencies of multiplicities $1,2, \ldots, 7$ are found to be $2722,1412,299,86,22,12$ and 2 . We also consider the distributions of periods of orbits and sub-orbits. For the even more restricted set of 474 systems with $V_{\mathrm{H}} \leqslant 4.00$ the proportions of higher multiples up to sextuple are progressively larger, suggesting incompleteness in even the relatively well-studied larger sample.

We construct a Monte-Carlo algorithm that will generate systems with roughly the observed multiplicities and orbital parameters, taking account of selection effects.
\end{abstract}

Keywords. stars: binaries, including multiples

\section{Introduction}

The Bright Star Catalogue (BSC: Hoffleit \& Jaschek 1983) is a fundamental resource when considering the stellar population of the Galaxy, or at any rate the nearer parts of the Galaxy. It lists multiplicities, but these are often visual multiplicities that may include line-of-sight, or 'optical', coincidences. Although roughly limited to magnitude 6.5 , it is not entirely complete to this magnitude, and also includes several fainter stars. The Multiple Star Catalog (MSC: Tokovinin 1997) carefully identifies many multiple systems, but restricts itself to multiplicity $\geqslant 3$. The Hipparcos Catalog (HIP: Perryman et al. 1997) has useful data such as parallaxes and proper motions that can help to distinguish optical from physical systems.

The great majority of multiple systems are 'hierarchical', with, for example, a wide 'binary' containing two closer 'binaries'. However non-hierarchical systems exist, in small numbers. We identify 17 non-hierarchical systems in our sample.

For 'brightness', we choose the Hipparcos magnitude system, and we include in the brightness all physical components even if their separation is quite wide. We make the cutoff at $V_{\mathrm{H}}=6.00$. The Hipparcos data are particularly useful because variable stars are averaged in a systematic way. A handful of stars which fail to be listed in the BSC, even down to magnitude 6.5, are Miras whose Hipparcos averaged magnitudes are brighter than 6.0 .

Note that we use the word 'system', and even the words 'multiple system', to include the possibility of multiplicity one, i.e., single stars; and we avoid the term 'star' as ambiguous. In this paper we count systems, and within systems we count components, so far as we are able. 


\section{Multiplicity}

In addition to the BSC, MSC and HIP we also used used the Eighth Spectroscopic Binary Catalog (BFM; Batten, Fletcher \& McCarthy 1989), the Ninth... (SB9; Pourbaix et al.2004), the Sixth USNO Catalog of Visual Binary Orbits (Hartkopf et al 2000), the $C C D M$ catalog (Dommanget \& Nys 2002) of close multiples (many of which however are optical rather than physical multiples), the GCVS (Samus et al. 2004) on eclipsers and ellipsoidal variables, and the CHARA survey of speckle observations (McAlister et al. 1989).

Several more catalogs have been scanned, either electronically or by eye. We do not list them here, for brevity, but they will be listed later in a more detailed publication. However, we mention one, Makarov \& Kaplan (2005), who considered systems whose Tycho and Hipparcos parallaxes and proper motions showed nonlinear behavior with time, i.e., acceleration, suggesting astrometric binaries. There are 349 such 'astrometric accelerators' in our catalog, denoted by 'A' in the reference column of Table 1. Occasionally one, or even more, is in a system already known to be a visual or spectroscopic binary, and we estimated whether the astrometric acceleration might or might not be due to the known companion.

In addition, several thousand papers were read, and are referenced directly if the data was different from (and, as we judged, better than) data from the principal catalogs mentioned above. Our overarching criterion was 'on the balance of probabilities', rather than 'beyond reasonable doubt'.

Following McClure (1983) and Boffin \& Jorissen (1988), we assume that all Ba stars are binaries, with a white-dwarf component. Many Ba stars have indeed been determined to have spectroscopic orbits, and in a small number a white dwarf has actually been detected in the UV. However the case for binarity is not just that some are confirmed binaries, but more strongly that a physical mechanism exists to explain the Ba anomaly in terms of binarity, specifically with a white-dwarf companion, and that the anomaly is very hard to explain otherwise. An example where we assume binarity, despite the absence of an orbit or a white-dwarf spectrum, is given in Table 1 (HR 459).

Table 1 lists a sample of our results. The main body of the Table is being prepared for electronic publication, and in the printed version here we include only a few examples, with a range of multiplicities. Many systems consist of two or more HR entries; we list them under the largest relevant HR number (Column 1). For 10 systems at the end which have no HR number, a 'pseudo-HR' number $(\geqslant 9201)$ is given, prefixed by ' $\mathrm{P}$ '. Column 2 lists what we consider to be the most reasonable multiplicity. Column 3 contains some reference letters (see below). Column 4 contains the parallax from Hipparcos. Column 5 contains our description of the configuration of the system using nested parentheses in roughly the format suggested by Evans (1977). For each individual component we give a magnitude and a spectral type, where we can find them, and for each pair of components we give either a period or a separation in arcseconds, where we can find them. Our reason for preferring this notation here is that, from experience, it can summarise a system sufficiently clearly that one can readily see where each component is in the hierarchy, and yet it only takes one line per system. To convey more information, both about the sub-components and the sub-orbits, the elegant notation of Tokovinin (1997) in the MSC is excellent, but this either takes $n-1$ lines for an $n$-tuple system, or else would be rather confusing to follow.

Reference letters in upper-case refer to particular catalogs, as listed in an annex to the on-line version. The absence of letters also indicates particular catalog sources: periods in days are from SB9, periods in years from USNO, spectral types from BSC, magnitudes (if 
Table 1. Sample Configurations of Bright Multiple Systems

\begin{tabular}{|c|c|c|c|c|}
\hline HR & $n$ & ref. & plx & configuration \\
\hline 4: & 1 & & 0.009 & $5.71 \mathrm{G} 5 \mathrm{III}$ \\
\hline 91: & 3 & & 0.002 & $5.55(5.95(\mathrm{~B} 5 \mathrm{IV}+? ; 27.80 \mathrm{~d}, \mathrm{e}=.20)+6.84 ; 152.7 \mathrm{y}, \mathrm{e}=.10)$ \\
\hline 120: & $2 ?$ & $\mathrm{~A}$ & 0.022 & $5.75(\mathrm{~F} 2 \mathrm{~V}+? ; ?)$ \\
\hline 136: & 6 & & 0.021 & $3.42\left(3.68\left(4.33\left(\mathrm{~B} 9 \mathrm{~V}+13.5 ; 2.4^{\prime \prime}\right)+4.55(\mathrm{~A} 2 \mathrm{~V}+\mathrm{A} 7 \mathrm{~V} ; 44.66 \mathrm{y}, \mathrm{e}=.74) ; 27.060^{\prime \prime}\right)+5.09\left(\mathrm{~A} 0 \mathrm{~V}+\mathrm{A} 0 \mathrm{~V} ; .1^{\prime \prime}\right) ; 540^{\prime \prime}\right)$ \\
\hline 152: & 2 & $\mathrm{R}$ & 0.005 & $5.26(\mathrm{~K} 5 \mathrm{III}+? ; 576.2 \mathrm{~d}, \mathrm{e}=.30)$ \\
\hline 165: & 3 & $\mathrm{R} A$ & 0.032 & $3.43\left((\mathrm{~K} 3 \mathrm{III}+? ; 20158 \mathrm{~d}, \mathrm{e}=.34)+13.0 \mathrm{M} 2 ; 28.7^{\prime \prime} \mathrm{bin}\right)$ \\
\hline 233: & 3 & G M & 0.004 & $5.47($ G8IIIa $+($ B9V $+? ; 226: \mathrm{d}, \mathrm{e}=.14:) ; 2091 \mathrm{~d}, \mathrm{e}=.53)$ \\
\hline 439: & 2 & $\mathrm{C}$ & 0.002 & $5.82\left(\mathrm{~K} 0 \mathrm{Ib}+\mathrm{B} 9 \mathrm{~V} ; .11^{\prime \prime}\right)$ \\
\hline 459: & $2 ?$ & & 0.006 & $5.58($ wd + K2IIIaBa; ?) \\
\hline 553: & 2 & $\mathrm{~s}$ & 0.055 & $2.60(\mathrm{~A} 5 \mathrm{~V}+\mathrm{G} 0: ; 107.0 \mathrm{~d}, \mathrm{e}=.88)$ \\
\hline 629: & $3 ?$ & $\mathrm{H} \mathrm{M}$ & 0.002 & $5.67\left((6.07 \mathrm{~B} 9 \mathrm{~V}+? ; ?)+6.95 \mathrm{~A} 1 \mathrm{Vn} ; 16.690^{\prime \prime}\right)$ \\
\hline 958: & 2 & R G s & 0.004 & $5.68(\mathrm{KOII}+\mathrm{A} 7 \mathrm{III} ; 115.0 \mathrm{~d})$ \\
\hline 1556: & 2 & $\mathrm{~s}$ & 0.006 & 4.74(WDA3 + S3.5/1-; ?) \\
\hline 1564: & 4 & $\mathrm{R} \mathrm{R}$ & 0.031 & $5.28\left((5.67 \mathrm{~F} 0 \mathrm{IV}+? ; ?)+6.59(\mathrm{~F} 4 \mathrm{~V}+? ; ?) ; 12.500^{\prime \prime}\right)$ \\
\hline 1788: & $6 ?$ & $\mathrm{~m} \mathrm{E} \mathrm{E}$ & 0.004 & $3.29\left(\left(3.58((\mathrm{~B} 1 \mathrm{~V}+\mathrm{B} 2 \mathrm{e} ; 7.990 \mathrm{~d}, \mathrm{e}=.01)+(\mathrm{B}:+? ; 0.864 \mathrm{~d}) ; 9.50 \mathrm{y}, \mathrm{e}=.2)+4.89 \mathrm{~B} 2 \mathrm{~V} ; 1.695^{\prime \prime}\right)+9.4 ; 115^{\prime \prime}\right)$ \\
\hline 2788: & $3 ?$ & M A E & 0.023 & $5.79((\mathrm{~F} 1 \mathrm{~V}+\mathrm{G} 8 \mathrm{IV}-\mathrm{V} ; 1.136 \mathrm{~d}, \mathrm{SD})+? ; ?)$ \\
\hline 4621: & $5 ?$ & M A A & 0.008 & $2.32\left(2.52(\mathrm{~B} 2 \mathrm{IVne}+? ; ?)+4.40(\mathrm{~B} 6 \mathrm{III} \mathrm{e}+? ; ?) @ 325^{\circ}, 267^{\prime \prime}+6.5 \mathrm{~B} 9 @ 227^{\circ}, 220^{\prime \prime}\right)$ \\
\hline 4908: & $2 ?$ & $\mathrm{~L}$ & 0.002 & $5.37\left(\mathrm{O} 9 \mathrm{Ib}+11.8 \mathrm{~K} 0 \mathrm{III} ; 29.1^{\prime \prime}\right)$ \\
\hline 5340: & 1 & $\mathrm{~s}$ & 0.089 & $0.11 \mathrm{~K} 1.5 \mathrm{IIIFe}-0.5$ \\
\hline 6046: & 2 & $\mathrm{R} \mathrm{As}$ & 0.005 & $5.77(\mathrm{M} 3 \mathrm{II}+\mathrm{G} 7 \mathrm{III} ; 2201 \mathrm{~d}, \mathrm{e}=.69)$ \\
\hline $7776:$ & 6 & $\mathrm{P}$ & 0.009 & $3.14\left(3.21\left((\mathrm{G} 8 \mathrm{II}+7.2(\mathrm{~B} 8 \mathrm{~V}+? ; 8.68 \mathrm{~d}, \mathrm{e}=.36) ; 1374 \mathrm{~d}, \mathrm{e}=0.42)+8.3 ; .8^{\prime \prime}\right)+6.09\left(6.16 \mathrm{~A} 0 \mathrm{III}+9.14 \mathrm{~A} 1 ; .68^{\prime \prime}\right) ; 205.3^{\prime \prime}\right)$ \\
\hline 8387: & 3 & $\mathrm{~m}$ & 0.276 & $4.83\left(\mathrm{~K} 4.5 \mathrm{~V}+\left(\mathrm{T} 1+\mathrm{T} 6 ; .73^{\prime \prime}\right) ; 402.3^{\prime \prime} \mathrm{bin}\right)$ \\
\hline 9072: & $1 ?$ & s & 0.031 & $4.12 \mathrm{~F} 4 \mathrm{IV}$ \\
\hline P9203: & $3 ?$ & $\mathrm{H} \mathrm{M}$ & 0.004 & $5.79\left(6.44(\mathrm{~B} 5 / 6 \mathrm{~V}+? ; ?)+6.67 \mathrm{~B} 8 / 9 \mathrm{~V} ; 129.490^{\prime \prime}\right)$ \\
\hline P9207: & 1 & & 0.007 & 5.99M8IIIvar \\
\hline
\end{tabular}

Note. -

1. The full Table will be placed on-line in due course, with a cross-reference Table for ID and a file of references.

2. For systems containing more than one HR component, we use the largest HR no. If there is no genuine HR no. in the system, we give a 'pseudo-HR' no., $\geq 9201$, prefixed by P. The corresponding HIP and/or HD numbers can then be found in the cross-reference Table. One example shown here, P9203, is HIP 32256 and 32269 . We identify only 9 pseudo-HR systems that qualify for our sample.

3. In col. 3, the letters refer to various sources, as mentioned in the text. The absence of a reference letter also implies particular catalog sources: BSC, HIP, MSC, SB9, USNO. 
quoted to 2 d.p.), separations (if quoted to 3 d.p.) and parallaxes from HIP. Lower-case reference letters $\mathrm{s}$ or $\mathrm{m}$ point to specific papers listed in the on-line version.

Table 2 gives the distribution over periods and spectral types. It is noticeable that the period distribution is severely bimodal at early types, becoming roughly unimodal by type F. The statistics on multiplicity are given later, Table 4, where they are compared with a theoretical model.

Table 2. Period Distribution in Systems and Subsystems

\begin{tabular}{|c|c|c|c|c|c|c|c|c|c|c|c|c|c|c|}
\hline $\log P(\mathrm{yr})$ & & -3.0 & -2.0 & -1.0 & 0.0 & 1.0 & 2.0 & 3.0 & 4.0 & 5.0 & 6.0 & 7.0 & 8.0 & total \\
\hline sp. & & & & & & & & & & & & & & \\
\hline O & 0 & 5 & 11 & 4 & 0 & 5 & 3 & 6 & 12 & 4 & 1 & 1 & 0 & 52 \\
\hline eB & 0 & 24 & 40 & 19 & 14 & 20 & 28 & 26 & 32 & 23 & & & 1 & 235 \\
\hline $\mathrm{IB}$ & 0 & 24 & 52 & 22 & 21 & 45 & 50 & 56 & 46 & 18 & ( & C & 0 & 340 \\
\hline A & 0 & 27 & 0 & 24 & 45 & 18 & $7 \varepsilon$ & 69 & 43 & 26 & & & 0 & 456 \\
\hline $\mathrm{F}$ & 0 & 14 & 30 & 24 & 38 & 49 & 68 & 38 & 24 & 14 & 2 & 1 & 0 & 302 \\
\hline G & 1 & 6 & 10 & 22 & 41 & 39 & 54 & 42 & 25 & 20 & 4 & & 1 & 266 \\
\hline K & 0 & 4 & 4 & 12 & 57 & 35 & 40 & 43 & 35 & 19 & 5 & & 1 & 255 \\
\hline M & 1 & 0 & 0 & 3 & 7 & 6 & 5 & 8 & 9 & 3 & : & & 0 & 45 \\
\hline sum & 2 & 104 & 208 & 130 & 223 & 277 & 326 & 288 & 226 & 127 & 31 & 6 & 3 & $\begin{array}{ll}3 & 1951\end{array}$ \\
\hline
\end{tabular}

Notes - The first column gives the spectral type of the dominant body in the system: eB means early B, i.e., B0 - B3.5, and IB means later B. Wolf-Rayet stars (2) are included under O; S and $\mathrm{C}$ stars (4) are included under M. The first column of integers gives the number of systems and sub-systems with $\log P(\mathrm{yr}) \leqslant-3.0$; the second for $-3.0<\log P \leqslant-2.0$, etc. The two shortest periods are a contact binary subsystem of the G dwarf 44 Boo (HR 5618), and a cataclysmic binary subsystem of the M giant CQ Dra (HR 4765). Long periods are estimates from the angular separation, distance, and Kepler's law.

\section{A Monte Carlo Model}

We attempt to model the data with a Monte Carlo procedure, based on that of Eggleton et al. (1989), selecting masses, mass ratios and periods from distributions with as few parameters as possible. We firstly draw a total system mass from a roughly Salpeter-like distribution, and then we divide it and sub-divide it according to a distribution of mass ratios, allowing a finite probability for the possibility that the mass ratio is zero, i.e., the system or subsystem does not in fact divide further. We allow a maximum of three subdivisions, so that the highest multiplicity we can obtain is 8 .

By postulating successive subdivisions, we do not intend to imply that actual multiple systems are produced by a physical mechanism of successive fragmentation. 'Successive bifurcation' is simply a convenient description of what hierarchical binaries actually are, regardless of how they are formed.

Let $X$ be a random number drawn from a uniform distribution on the interval $[0,1]$. If we take the system mass to be $M=f(X)$, we are in effect postulating a distribution such that $X(M) \equiv f^{-1}(M)$ is the normalised cumulative distribution. The distribution given by

$$
M=M_{0} \frac{X^{\alpha^{\prime}}}{(1-X)^{\alpha}},
$$

has median $M_{0} 2^{\left(\alpha-\alpha^{\prime}\right)}$, and is roughly Salpeter-like at large $M(X \sim 1)$ if $\alpha \sim 0.75$.

Our attempts to model the distribution of bright systems will clearly not cast any light on the distribution of K/M dwarfs, since hardly any appear in Table 1 except as 
secondaries or tertiaries. Consequently we adopt the simplification that $\alpha^{\prime}=\alpha$, i.e., that the distribution is symmetrical (in $\log M$ ) about the median, which we expect to be at $M_{0} \sim 0.3-0.5$, appropriate to early $\mathrm{M}$ dwarfs. We adopt provisionally $M_{0}=0.30 M_{\odot}$, $\alpha=0.83$.

The bifurcation probability is determined by another $X$, a new selection from the random-number generator. We choose a function $X_{\mathrm{bf}}(l, M)$ defined on the interval $[0,1]$, and require that the system bifurcate at hierarchical level $l$ if $X \leqslant X_{\mathrm{bf}}$, and not bifurcate if $X>X_{\mathrm{bf}}$. We supply $X_{\mathrm{bf}}$ in tabular form, using as independent variable

$$
m=5+2 \log \left(\frac{1+100 M}{100+M}\right)
$$

Of course, the bright-system sample tests the algorithm seriously only for $M \gtrsim 1 M_{\odot}$ $(m \gtrsim 5)$, i.e., the 15 values at the right.

Table 3. Bifurcation Probability $X_{\mathrm{bf}}$ as a Function of Mass and Hierarchical Level

$\begin{array}{rrrrrrrrrr}\mathrm{m}= & 1 & 2 & 3 & 4 & 5 & 6 & 7 & 8 & 9 \\ \mathrm{M}= & 0 & .01 & .09 & .32 & 1 & 3.2 & 11 & 32 & \infty \\ \mathrm{l}=0 & 0.50 & 0.50 & 0.50 & 0.50 & 0.50 & 0.65 & 0.80 & 0.87 & 0.92 \\ 1 & 0.10 & 0.15 & 0.15 & 0.15 & 0.15 & 0.15 & 0.20 & 0.45 & 0.65 \\ 2 & 0.00 & 0.00 & 0.00 & 0.00 & 0.00 & 0.20 & 0.50 & 0.70 & 0.85\end{array}$

The periods are chosen from further distributions, which at the highest hierarchical level $(l=0)$ we take to be somewhat similar to $(1)$ :

$$
P=P_{0} \frac{X^{\beta^{\prime}}}{(1-X)^{\beta}} .
$$

We adopt $P_{0} \sim 270$ yrs, $\beta=2.5, \beta^{\prime}=2.0$.

For subsystems or subsubsystems, if any, we choose a period ratio, relative to the next level up, of

$$
\frac{P^{\prime}}{P}=A \cdot 10^{-\gamma X}
$$

We provisionally adopt $A=0.2, \gamma=5$.

The mass ratio $q$ at each bifurcation, if it occurs, is taken from another distribution:

$$
q=\max \left(0.01, X^{\delta}\right),
$$

where the exponent $\delta$ might in principle be a function of both $M$ and $P^{\prime}$. We provisionally adopt $\delta=0.8$. We disallow $q<0.01$.

For the present we assume that all orbits are circular. It would not be difficult to generate a distribution of eccentricities, but we feel that there are too many wide orbits, of unknown eccentricity, to make this refinement necessary at present.

The age is chosen from a very simple distribution:

$$
t=t_{0} X
$$

where $t_{0}$ is an estimate of the age of the Galaxy, $t_{0} \sim 10^{10} \mathrm{yr}$. This assumes a uniform production-rate of systems. It is unlikely to be a good assumption over the whole range of possible ages, but most of the systems in Table 1 are massive enough that they must have been formed in the last $10 \%$ of the Galaxy's lifetime, and so the assumption of constancy may not be very critical.

The evolution is treated so far in a rather cursory manner, which we hope to improve on in a future paper. In particular, we ignore such binary-specific evolutionary processes as Roche-lobe overflow (RLOF), although we note systems in which we can expect it to 
have happened. We also ignore the fact that neutron stars are typically blasted out of their parent system by a slightly anisotropic supernova explosion; thus many multiples that we create contain one or more neutron stars. But for the present we evolve each component independently according to its mass and age, following roughly the simple procedure of Eggleton et al.(1989).

Finally, we need to generate a distribution over distance $d$. Given a set of component masses, as above, we can evolve them to the selected age and thus determine (a) the total luminosity, and (b) the distance $d_{\max }$ out to which the system will be visible above the set limit $\left(V_{\mathrm{H}}=6\right.$, say). Suppose that we generate a total of $N=10^{6}$ systems. To a first approximation, they might populate uniformly a sphere of radius $D \sim 100 \mathrm{pc}$. We can think of the given system as being 'cloned' $n \equiv\left(d_{\max } / D\right)^{3}$ times, with the $r$ th clone placed randomly within a spherical shell of inner and outer radii $(r-1)^{1 / 3} D$ and $r^{1 / 3} D$, i.e., it would be at distance

$$
d_{r}=\left(r-1+X_{r}\right)^{1 / 3} D, r=1,2, \ldots, n,
$$

where the $X_{r}$ are a sequence of independent random numbers in [0,1]. Of course, if $d_{\max }<D$ the system will only be seen at most once, with probability $\left(d_{\max } / D\right)^{3}$; but that is in fact what Equation 7 implies with $n=1$. Since we have defined $D$ for $10^{6}$ systems, we can write $D=10^{2} d_{\text {nbr }}$ where $d_{\text {nbr }}$ is the mean distance between nearest neighbors. We adopt $d_{\mathrm{nbr}}=1.39 \mathrm{pc}$.

To model a more realistic density distribution (that does not experience Olbers' paradox), suppose that the distance to the $r$ th clone is

$$
d_{r}=x^{1 / 3} D \sqrt{1+x^{1 / 3} D / h}, x=r-1+X_{r} .
$$

This approximation is roughly what we expect for a uniform distribution within a disk of thickness $h$. We make the vertical scale-height $h$ age-dependent, $h=200 t^{0.3}$, with $h$ in $\mathrm{pc}$ and $t$ in units of $10^{10} \mathrm{yr}$.

A further small refinement that we put in is interstellar absorption, so that the apparent luminosity $l$ of a system at distance $d$ whose intrinsic luminosity is $L$ will be given as

$$
l=\frac{L}{d^{2}} e^{-k d},
$$

with $k \sim 0.001$ ( $d$ in parsecs), corresponding to roughly 1 magnitude per kiloparsec. This means that the total number of observable clones will in practice be less than the crude number $n$ estimated above.

We put in one further procedure which is convenient numerically though it has no astrophysical significance. A given component is at its brightest, as a giant, for only a short period of time compared with the Galactic age. Given a mass and age selected at random as above (and considering, for the purpose of exposition, only single stars) the chance is rather small, even among $10^{6}$ samples, that a given star will be in this rather bright stage; but on the other hand it will require to be cloned many times since it will be visible to a large distance. This introduces considerable scatter as between one simulation and another that is identical except insofar as the random numbers differ. We can mitigate this by using a non-uniform distribution of $X, Y$ over the unit square, and using the Jacobian of the transformation to modulate the density. Thus the sample of $10^{6}$ systems, which might produce only say 10 giants cloned 100 times each, can produce instead say 500 giants cloned 2 times each.

Once we have a Monte Carlo selection of multiples, we can then examine them in a 'theoretical observatory', to determine how many of the components and sub-binaries will be actually recognisable. We look for spectroscopic binaries, assuming that the velocity 
amplitude has to be greater than some threshold which we take to depend mainly on spectral type: fairly low for G/K components $(\sim 2 \mathrm{~km} / \mathrm{s})$, increasing to $\sim 30 \mathrm{~km} / \mathrm{s}$ for O components, which tend to have both few and broad lines, and also to be intrinsically variable in radial velocity at this level. We further required the period to be less than 30 years, to simulate the patience of observers.

For a visual binary to be theoretically observable, we require that the angular separation be greater than some threshold that depends on the difference in magnitude of the (sub)components:

$$
\rho>0.4\left|\Delta V_{\mathrm{H}}\right|+0.1
$$

where $\rho$ is the angular separation in arcsecs. We further require that the combined magnitude of a detectable component or subsytem be $\leqslant 14$. Although we can expect that there are many companions that are fainter, Table 1 has only a handful fainter than this limit.

Table 4. Multiplicities in Theoretical and actual Samples

\begin{tabular}{rrrrrrrrrrr}
\hline & total & 1 & 2 & 3 & 4 & 5 & 6 & 7 & 8 & av. \\
\hline$V_{\mathrm{H}} \leqslant 6$ & & & & & & & & & & \\
raw & 4649 & 1596 & 2041 & 531 & 285 & 118 & 43 & 31 & 4 & 2.05 \\
apparent & 4649 & 2918 & 1323 & 279 & 93 & 24 & 9 & 3 & 0 & 1.50 \\
actual & 4555 & 2722 & 1412 & 299 & 86 & 22 & 12 & 2 & 0 & 1.53 \\
$V_{\mathrm{H}} \leqslant 4$ & & & & & & & & & & \\
raw & 566 & 178 & 256 & 60 & 43 & 16 & 9 & 3 & 1 & 2.13 \\
apparent & 566 & 313 & 189 & 43 & 13 & 4 & 2 & 2 & 0 & 1.62 \\
actual & 474 & 213 & 176 & 52 & 18 & 9 & 6 & 0 & 0 & 1.84 \\
\hline
\end{tabular}

\section{Discussion}

The result of the above selection effects is to transform the raw multiplicities into apparent multiplicities. Table 4 is an example, for both the larger sample of $V_{\mathrm{H}} \leqslant 6$ and the smaller sample $V_{\mathrm{H}} \leqslant 4$ : the top row gives the raw multiplicities, the second row the apparent multiplicities after theoretical observation, and the third row the observed multiplicities. It can be seen that in the larger sample the frequencies of multiplicities 1 to 7 are reasonably matched, with the main discrepancy being a $\sim 6 \%$ shortage of binaries in the 'apparent' sample. The overall average multiplicity is reasonably matched within the limits of small-number statistics. For the smaller sample there is less good agreement in the average, although it has moved in the right direction. This discrepancy, although fairly modest, confirms our view that the smaller sample has simply been more thoroughly studied than the larger. Our model, however, is based on trying to fit the larger sample (including the data of Table 2), because of its greater statistical weight.

The bimodality of the period distribution at early types seen in Table 2, shading to unimodality at later types, is in fact quite well-modeled by our 'apparent' data. This supports, though it does not prove, the view that it is largely a selection effect.

Data on multiplicity is important as a constraint on (a) the star-formation problem, (b) the problem of the evolution of the Galactic stellar population, (c) $N$-body simulations of dynamics and evolution of clusters and (d) the interaction of dynamics and evolution through the effect of Kozai cycles. We discuss one sub-topic each of (b), (d) briefly.

A likely effect of triples under (b) is the production of 'anomalous binaries'. In shortperiod binaries we can expect that a merger of the two components is a fairly common event. Case A systems can evolve conservatively only if the initial mass ratio is fairly 
mild ( $q \gtrsim 0.6$; Nelson \& Eggleton 2001), and if $q$ is not this mild then a merger seems quite a likely event. It would be hard to determine that a particular currently-single star is a merged remnant of a former binary. But within a primordial triple system, it is possible that such a merged remnant would be identifiable, because the wide binary that remains after the merger of the close sub-binary would be expected, in at least some circumstances, to show an anomaly where the two components appear to be of different ages. R.E.M. Griffin (to be published) has found a number of such apparently anomalous systems, of which $\gamma$ Per (HR 915) is an example. Although the mass ratio, obtained from careful deconvolution of the two spectra (G8III $+\mathrm{A} 2 \mathrm{IV} ; 5330 \mathrm{~d}, e=0.79$ ), is $1.53\left(M_{G} / M_{A}\right)$, the A component seems surprisingly large and luminous compared with what it should be on the ZAMS; and it ought to be very close to the ZAMS if it is coeval with the $\mathrm{G}$ component. A possible explanation is that the giant is the merged product of a former sub-binary with a mass-ratio of $\sim 0.5$, since this could allow the more massive two of the original three components to evolve at roughly the same rate (Eggleton \& Kiseleva 1996, Eggleton 2006). We hope to test shortly the possibility that the appropriate primordial triple parameters, from our Monte Carlo model, will give an acceptable number of potential progenitors. Alcock et al. (1999) and Evans et al. (2005) have noted that a similar process might lead to Cepheid binaries of an anomalous character, such as may be required to reconcile observed Cepheids with the theoretical models of the Cepheid pulsation phenomenon.

Under (d), triple stars in which the two orbits are misaligned can be subject to the dynamical effect of Kozai cycles, and these in turn can allow tidal friction to become important in the course of $10^{6}-10^{9}$ yrs and cause the inner orbit to become smaller. Tokovinin \& Smekhov (2002) have noted that periods above $\sim 10$ days are rarer (relative to shorter periods) among the inner binaries of triples than in the population as a whole, and possibly this is accounted for by the combination of Kozai cycles and tidal friction (KCTF). Pribulla \& Rucinski (2006) have noted that as many as $42 \%$ of contact binaries appear to be in triples (and arguably $59 \%$ in a more thoroughly examined subsample), and it could be that KCTF has contributed to this; although we probably need the additional influence of magnetic braking to drive fairly close low-mass binaries generated by KCTF to contact on a timscale of $\lesssim 10^{9}$.

\section{Acknowledgements}

This study has been carried out partly under the auspices of the U.S. Department of Energy, National Nuclear Security Administration, by the University of California, Lawrence Livermore National Laboratory, under contract No. W-7405-Eng-48.

\section{References}

Alcock, C. et al. (1999) AJ, 117, 920

Batten, A.H., Fletcher, J.M., \& McCarthy, D.G. (1989; BFM) PDAO, 17, 1

Boffin, H.M.J. \& Jorissen, A. (1988) A\&A, 205, 155

Dommanget, J. \& Nys O. (2002; CCDM) Observations et Travaux 54, 5

Eggleton, P.P. (2006) in A Life with Stars, eds. Kaper, L., van der Klis, M., \& Wijers, R.A.M.J., New Astron. Rev., in press

Eggleton, P.P., Fitchett, M.J., \& Tout, C.A. (1989) ApJ, 347, 998

Eggleton, P.P. \& Kiseleva, L.G. (1996) in 'Evolutionary Processes in Binary Stars' ed. Wijers, R. A. M. J., Davies, M. B., \& Tout, C.A., NATO ASI Series C, 477, p345

Evans, D.S. (1977) Rev. Mex A\&A, 3, 13

Evans, N.R., Carpenter, K.G., Robinson, R., Kienzle, F., \& Dekas, A.E. (2005), AJ, 130, 789

Hartkopf, W.I., Mason, B.D., McAlister, H.A., et al. (2000; USNO) AJ, 119, 3084 
Hoffleit, D. \& Jaschek, C. (1983; BSC) The Bright Star Catalogue, $4^{\text {th }}$ ed. New Haven: Yale University Observatory

McAlister, H.A., Hartkopf, W.I., Sowell, J.R., et al. (1989; CHARA) AJ, 97, 510

McClure, R.D. (1983) ApJ, 268, 264

Makarov, V.V. \& Kaplan, G.H. (2005) AJ, 129, 2424

Nelson, C.A. \& Eggleton, P.P. (2001) ApJ, 552, 664

Perryman, M.A.C., Lindegren, L., Kovalevsky, J., et al. (1997; HIP) A\&\&A, 323, 49

Pourbaix, D., Tokovinin, A.A., Batten, A.H., et al. (2004; SB9) A\&SA, 424, 727

Pribulla, T. \& Rucinski, S.M. (2006) AJ, 131, 2986

Samus, N.N., Durlevich, O.V. et al., (2004;GCVS) Institute of Astronomy of Russian Academy of Sciences and Sternberg State Astronomical Institute of the Moscow State University

Tokovinin, A.A. (1997; MSC) A\&AS, 124, 75

Tokovinin, A.A. \& Smekhov, M.G. (2002) A\&A, 382, 118

\section{Discussion}

JuAn Manuel EchevarRia: From your sample, which is close to the number of stars seen by the naked eye (at least of an 18 year old person), what is the percentage of multiple stars in the 'classical' sense (i.e., 2 or more)?

EgGLETOn: The proportion of 2 or more is about $40 \%$ for the 6 th mag. sample, and about $55 \%$ for the 4 th mag. subsample. In our theoretical 6 th mag sample, before theoretically observing them, the proportion is about $65 \%$.

Theo Ten BrummelaAR: You seem to have a large number of free parameters to fit what seems to be a rather small sample. Could you please comment on this?

EGgleton: There are about 20 parameters that matter. We consider this to be quite a small number considering the range of multiplicities we attempt to encompass. Supposing that one could divide about 5000 stars into 20 bins, each bin being dominated by one parameter, we might reasonably hope that the parameters could be individually estimated to $10 \%$. Of course in the real world we would find some parameters less tightly constrained and some more tightly.

Roger GRIfFIn: I think you may have inflated your figures for multiplicity by your 'balance of probability' criterion. In the case of HR 233 you particularly commented on the period of 226 days for the inner orbit. That period does not exist - the orbit concerned was withdrawn by the author and repudiated by the editor of the journal concerned (see the first page of the May 1984 Astronomical Journal).

EGGLETON: I am aware of the inadequacy of that particular claim. However R.E.M. Griffin tells me that nevertheless the hot star appears to have greater r.v. variation, and on a shorter timescale, than is likely for just a binary with your well-established longer period. I think it is therefore still reasonable to conclude on a balance of probabilities that the system is at least triple. I hope you will be pleased to see that Arcturus (HR 5340) is listed by us as probably single, although Hipparcos claimed to resolve it into 2 . We noted your analysis $(O b s, 118,299,1998)$ that showed that the very accurate radial velocities over $\sim 50$ years do not support such binarity. 